\title{
Ethnographically Derived Socio-technical Analysis for Information System Support in Intensive Home Care
}

\author{
Markus Jelonek ${ }^{*}$, Thomas Herrmann, Michael Ksoll, and Nina Altmann \\ Ruhr University Bochum, Universitätsstraße 150, 44780 Bochum, Germany \\ \{markus.jelonek, thomas.herrmann, michael.ksoll, \\ nina.altmann-z49\}@rub.de
}

\begin{abstract}
Developing information systems and collaboration support in the field of intensive home care is faced with challenging conditions of empirical work for requirements elicitation. On the one hand, the huge variety of aspects and situation-related characteristics require a deliberate analysis and close interaction with caregivers and relatives to identify the needs and constraints of technical support. On the other hand, the relevant places are hardly accessible. To be able to visit and observe the clients in situ, consent is needed by the caregiver, the client, and the relatives living with them, and a high level of privacy and confidentiality has to be maintained. Based on an ethnographical data gathering and analysis, this article provides a comprehensive categorization that characterizes the work of caregivers in intensive home, to make the work of information system designers in this field more efficient: activities, interpersonal interaction, documentation, qualification, interaction with technology, client autonomy, history of medical records, and feedback on intensive home care. Finally, these categories are compared with related work of socio-technical design of health care information systems.

Keywords: Health Care, Intensive Home Care, Information System Design, Grounded Theory, Ethnography, Socio-technical Requirements.
\end{abstract}

\section{Introduction}

Developing information systems (IS) for supporting intensive health care at home implies challenging conditions that have to be taken into account by IS designers. Work in this area is largely value driven [1], [2] and has to be sensitive with respect to a number of specific characteristics [3]:

- The clients ${ }^{\dagger}$ cannot survive without continuous assistance.

\footnotetext{
* Corresponding author
}

(C) 2020 Markus Jelonek et al. This is an open access article licensed under the Creative Commons Attribution License (http://creativecommons.org/licenses/by/4.0).

Reference: M. Jelonek, T. Herrmann, M. Ksoll, and N. Altmann "Ethnographically Derived Socio-technical Analysis for Information System Support in Intensive Home Care," Complex Systems Informatics and Modeling Quarterly, CSIMQ, no. 22, pp. 1-20, 2020. Available: https://doi.org/10.7250/csimq.2020-22.01

Additional information. Author ORCID iD: M. Jelonek - https://orcid.org/0000-0003-1434-2952, T. Herrmann https://orcid.org/0000-0002-9270-4501. PII S225599222000126X. Received: 30 November 2019. Accepted: 12 February 2020. Available online: 30 April 2020.

\footnotetext{
$\dagger$ As common for intensive home care, we refer to persons receiving care as "clients" [4].
} 
- Different caregivers have to be present in the clients' home during different shifts at 24 hours a day; the clients' relatives must be able to rely on them. Thus, information and experience exchange has to take place between the caregivers.

- There is only a loose coupling between the caregivers and their employer since a caregiver usually spends nearly the entire working time at the same place.

- Compared to ambulatory care, the caregivers' work can include a lot of idle time when they are only present and ready in the case of emergencies. This situation implies a strongly varying stress level.

- Caregivers and the relatives have to handle a series of coordinative problems of task sharing.

- Being present in a private home all day establishes a specific social situation.

- Care work in this context cannot be completely rule based [5].

IS design has to consider these specific conditions when developing technology to support information processing and exchange. To understand the requirements for information exchange in intensive home care, on the one hand, it would be feasible to carry out deliberate ethnographical analysis (e.g. [5], [6]) and to pursue a participatory design approach where users and the stakeholders are systematically involved in cycles of design and testing. On the other hand, a project to identify potential use cases of augmented reality glasses [7] for caregivers revealed that the access to the field of intensive care is hindered by several obstacles and includes a lot of organizational effort and unpredictable waiting time. Roughly, the reasons are:

- Consent is needed by relatives, clients and the care service to grant access for observations and interviews, and involving an ethical committee is mostly indispensable.

- The clients' physical and psychological conditions can restrict their capability to describe their situation, or limit the opportunities where they can be observed.

We have experienced these difficulties when managing to get access to 21 interviews and 10 observations (at 9 different clients' home environments) during 8 months in 2016 and 2017. From this experience, we conclude that requirements for information system support in the field of intensive home care cannot be done efficiently and timely when relying on ethnographic analysis and participatory design in every case. Therefore, insights from our study should help to develop guidelines and solutions in the field of intensive home care.

The background for this exhaustive analysis is a project that investigates the potential benefits of applying smart glasses for information capturing and displaying, especially in situations where people need their hands to handle certain tasks. Clearly, the smart glasses are not the only devices that can be used to process information (e.g. [8], [9], [10]). For instance, the user can collect information via speech recognition and the glasses provide feedback whether the recognition is successful, but later on, the collected information is displayed on tablets etc. We learned that developing solutions in this area has to pursue a socio-technical approach [11] where technical and organizational solutions have to be brought together. For instance, we can imagine a user story such as:

"As a caregiver I want to maintain and refer to a document of agreements between me and the relatives about task sharing."

Firstly, this exemplary requirement is based on confidential information given by interviewees that conflicts occurred between caregivers and relatives about sharing specific tasks. For instance, caregivers are not authorized to carry out housekeeping tasks but sometimes do so to maintain a practicable relationship with the relatives. Secondly, with respect to the solution, it is not appropriate to store such a document in an information system since taking over housekeeping tasks is not an official part of the contract of caregiver services. Insurance problems are involved etc. Therefore, such a list of agreements might be dictated by speech but can only be ephemerally stored, but to be documented on paper. Obviously, technical support 
and a kind of organizational rule are combined here. From this example, we learn that the kind of a requirement itself and the possible solutions are influenced by confidential information and close insights that should be accessible to requirements engineers in this field. Thus, we propose our ethnographically derived requirements that consist of categories and sub-categories describing the specific situation of intensive home care. For each category, an abstract description is provided as well as enriching and illustrative examples.

In what follows, we refer to related work, outline the methodological approach and provide the main categories that help to understand the work and needs in the interplay of caregivers, clients and relatives. Additionally, we compare our results with generalized hints and conditions for socio-technical design of health care systems. These generalized hints are presented as a concluding chapter derived from 10 case studies in health care [5]. Finally, the conclusion emphasizes the main aspects to be mirrored by the requirements to be met by information systems in this field.

\section{Related Work}

The demographic development in Germany and many other countries around the world results in a lack of trained medical staff and an increasing proportion of an elderly population. Therefore the demand for technology to support (health-)care [12] has increased, as well as for the integration of informal caregivers in home care [13], [14], thus shifting institutional care to care settings at home [15] with an increasing relevance for the future. Although there is little scientific research about the requirements for information systems in intensive home care, many publications can be found about intertwining areas like nursing homes, domestic home care for the elderly, dementia care homes or nursing in general [e.g. 16]. Here, much scientific work has been done by using an ethnographic approach to explore the field of work. Publications in this area range from, for instance, the subjective experiences of informal caregivers in home care [18] to the support of work in home care for the elderly by using collaborative technologies for note sharing and other purposes [16].

Due to the nature of the domain of home care settings, a privacy-sensitive procedure in eliciting comprehensive requirements is needed. As described in other works, designing technologies for home care needs to address collaboration between different actors in the care settings [16], [17]. Recent research within the Computer Supported Cooperative Work and Human-Computer Interaction (CSCW/HCI) view focuses on the support of individuals and cooperative work by using an ethnographic approach to open up the fields of interest. The use of the Grounded Theory Method offers a way to explore a domain with the emphasis on discovery of new insights and building up a partial understanding of the context [19]. Its use has increased in HCI to explore implications for socio-technical requirements and therefore, efforts have been made, how to integrate ethnographic research in the process of requirements elicitation [6], [8], [20].

One of the early examples that linked ethnographic methods with the identification of social organization in cooperative work can be found in the work of Hughes, Randall and Shapiro [21] in a project on air traffic control, in which they discussed the electronic representations of manual practices based on their ethnographic findings. Ethnographic fieldwork has since been used in a variety of studies, for instance, to analyze the settings of domestic home care and the work done by elderly informal caregivers [22]. Especially in the field of health care, the requirements engineering process challenges researchers with no background in health care, for instance, how the used vocabulary for the domain has to be understood [23]. Another example that addresses the complexity of the health care domain focused on requirements for mobile electronic health care records, resulting in a framework outline which was based on observational studies performed in a hospital [24]. For home care technology in general, an exploratory study suggests a participatory elicitation and negotiation of requirements [25]. 
We suggest combining an ethnographic approach with the socio-technical analysis of requirements [26]. The $i^{*}$ modeling method is a striking example of how social aspects can be taken into account and combined with the development of information systems [27]. However, while $i^{*}$ [28] emphasizes the "why?" of the involved roles' or actors' behavior (by modeling hard and soft goals), ethnography emphasizes the observation of what is going on and how. In 2011, Baxter \& Sommerville [11] summarized the relevant socio-technical approaches with respect to systems engineering. A recently edited collection of case studies in sociotechnical design of health care systems [5] provides a series of important aspects to be taken into account [29]. Ackerman's et al. [30] case study on home-based care for people with spinal injuries provides a helpful example that allows to point out differences to be considered if professional home care is conducted all around the day as in the case of intensive home care. A detailed consideration of Ackerman's et al. conclusions [29] is provided in Section 5.

\section{Method}

We used a qualitative approach for the data collection. This was motivated by the fact that the described field of intensive home care has not yet been studied in detail and that the chosen method is well established for gaining information about unknown research fields. Using Grounded Theory (GT), our aim was to provide insights about this field based on our collected data to answer our research question. From this approach, we expect that interested researchers can get insights in the field of intensive home care and are able to derive extensive descriptions for possible information system solutions. In the following sections, we describe how we collected and analyzed data (Figure 1). We used open and axial coding suggested by GT to generate a category system to get an overview of the field.

\subsection{Data Collection}

To derive an insightful description of the domain that includes rich and concrete examples, our ethnographical research procedure (Figure 1) is based on the inductive analysis approach of the GT by Corbin \& Strauss [31]. Our sample is composed of 17 employees of various care service providers and 4 relatives of intensive home care clients. For the data gathering, we applied triangulation of data [32] as the interviewed caregivers come from different cities in Germany, were contacted via various care service providers and the interviews were partly combined with observations inside the clients' environments. In total, we conducted 21 interviews, drew up 10 observation logs, interviewed 17 professional caregivers and 4 relatives of different clients.

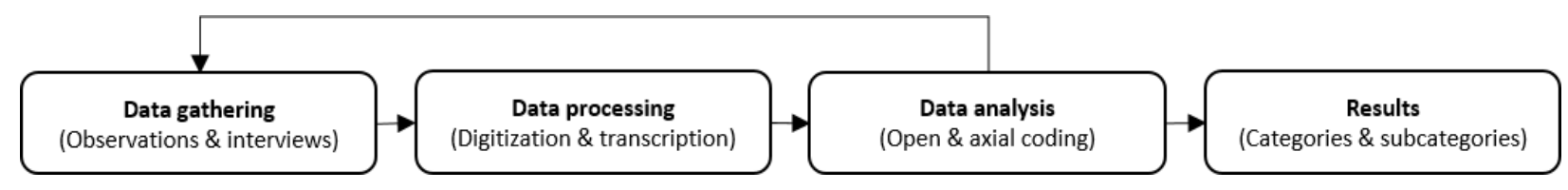

Figure 1. Applied ethnographical research process

The data was collected between July 2016 and February 2017 by three different researchers. Interviews were conducted by a semi-structured guideline which focused mainly on questions regarding the procedures of daily care routine to understand the characteristics and conditions of intensive home care. Our interviews ranged between 20-130 minutes. Each interviewee filled out a short questionnaire to gather sociodemographic information prior to the interviews. In our resulting sample, the interviewees' ages ranged from 25 to 72 years. Two out of 21 were male interviewees. Eight out of 17 professional caregivers were qualified as caregivers for the elderly. The remaining nine caregivers were qualified in nursing. 


\subsection{Data Analysis}

For analyzing and interpreting the data we transcribed the recorded interviews via a basic transcript system by Kuckartz et al. [33] and used the procedures of open and axial coding suggested by GT as instruments to create categories on the conducted ethnographic material [31], [34]. First, we used open coding to break data apart and to generate preliminary categories. Later, the axial coding procedure was used to systematically examine the relations between our categories and afterwards between categories and subcategories. It has to be taken into account that open and axial coding are separated analytical procedures, but go hand in hand during the data analysis [31]. For the data coding we used the software MAXQDA ${ }^{\ddagger}$. The data analysis aimed on condensing our findings into a category system that is feasible to guide information system designers in the field of intensive home care in general.

Initially, a group of three (en-)coders started with open coding of one interview with a caregiver and one observation log. Every coder worked with the same two documents. We used constant comparison and questioning to examine our data [31]. After this first iteration, we compared our coding results and agreed on using short and concise terms for the concepts. In addition, we decided that coded paragraphs must be self-explanatory to be able to draw conclusions from the context for further data investigation. Subsequently, we coded two additional documents (one observation log and one interview with a caregiver) by open coding again to test if our developed coding approach, we agreed upon before, worked for us as intended. The open coding procedure resulted in 212 preliminary concepts in total. Due to this huge number of concepts, we merged those ones with semantic similarities. According to the axial coding procedure, we looked at the relations between the different concepts and also matched and combined concepts to superior codes. Using this procedure, we were able to develop a preliminary category system, including a coding guideline, which we tested on further two interviews with clients' relatives. Based on the new results we revised and adjusted our preliminary categories. Finally, we created a detailed coding guideline, which consists of the categories' terms, content descriptions for the main categories and subcategories, rules for the application of the categories, examples for the application from the raw data and hints for the delineation of other categories [34]. We tested our coding guideline on the initial interviews and observation logs and on two new documents, which got peer-reviewed by a health care expert. Altogether, we coded eight documents until we developed our final category system and coding guideline that we used for coding and analyzing each of the 21 interviews and 10 observation logs (Table 1). The complete category scheme consists of eight categories and 47 subcategories in total, whereas the number of subcategories is different for each category.

Through this elaborate analysis, the categories, with their subcategories, offer precise descriptions of the work in home intensive care. As example and to describe the categorization in more detail the subcategories of the category "Documentation" are listed in Table 2. This example shows that a total of five codes could only be assigned to the main category "documentation" and not to any of the subcategories. Such cases rarely occur, for instance, when data related to documentation has been coded, but did not fit into any of the subcategories and no new category could be created from it.

Finally, detailed descriptions are offered to help understand how the code scheme can be applied. These descriptions cover the content, the application and a coding example. For instance, the details for the subcategory "Information about clients" refer to the documentation of client-specific needs and wishes, or information about illnesses (Table 3).

\footnotetext{
${ }^{*}$ https://www.maxqda.com/
} 
Table 1. Categories and code distribution

\begin{tabular}{|l|r|r|}
\hline \multicolumn{1}{|c|}{ Category } & Number of codes & \multicolumn{1}{c|}{$\%$} \\
\hline Activities in intensive home care & 594 & 26.87 \\
\hline Interpersonal interaction & 572 & 25.87 \\
\hline Documentation & 272 & 12.30 \\
\hline Qualification & 87 & 3.93 \\
\hline Interaction with Technology & 180 & 8.14 \\
\hline Client Autonomy & 101 & 4.57 \\
\hline History of medical records & 60 & 2.71 \\
\hline Feedback on intensive home care by relatives and caregivers & 345 & 15.60 \\
\hline & 2211 & 100.00 \\
\hline
\end{tabular}

Table 2. Code distribution for Category "Documentation"

\begin{tabular}{|l|r|r|}
\hline \multicolumn{1}{|c|}{ Category and Subcategories } & Number of codes & \multicolumn{1}{c|}{$\%$} \\
\hline Documentation & 5 & 1.84 \\
\hline - Information about clients & 80 & 29.41 \\
\hline - Necessity of documentation & 37 & 13.60 \\
\hline - Documentation Issues & 22 & 8.09 \\
\hline - Content and scope of documentation & 103 & 37.87 \\
\hline - Documentation effort & 25 & 9.19 \\
\hline & 272 & 100.00 \\
\hline
\end{tabular}

Table 3. Details for the Subcategory "Information about clients"

\begin{tabular}{|c|c|}
\hline Content & $\begin{array}{l}\text { Describes the documentation of client-specific preferences, information about } \\
\text { illnesses, etc. for the current care situations (from the beginning of the intensive } \\
\text { home care). }\end{array}$ \\
\hline Application & Use, if preferences or illnesses of the client are documented. \\
\hline Example & $\begin{array}{l}\text { Then [caregiver] SRH19 goes to the nursing room to get the insulin schedule } \\
\text { from the doctor (from the documentation folder) to see how many syringes need } \\
\text { to be injected. }\end{array}$ \\
\hline
\end{tabular}

Here, a differentiation to the category "Biography of clients" (subcategory of "History of medical records") is noticeable (Table 4). Historical illnesses would be categorized with the category "Biography of clients" as it is part of his/her life prior to intensive home care, whereas illnesses with the beginning of the intensive home care could be categorized as "Information about clients". However, this approach of categorization remains subjective and semantic overlapping of categories is possible. 
Table 4. Details for the subcategory "Biography of clients"

\begin{tabular}{|l|l|}
\hline Content & $\begin{array}{l}\text { Describes information on the history of illness, life events of the client that are not } \\
\text { part of the care documentation. }\end{array}$ \\
\hline Application & $\begin{array}{l}\text { Use, for instance, when the illness history of the client or prior life circumstances } \\
\text { are described. }\end{array}$ \\
\hline Example & $\begin{array}{l}\text { Interviewer: And [to this time] you also had [the possibility to ask] a care service } \\
\text { provider? } \\
\text { PBH22: That came much later, that's only five years ago, that I had early morning } \\
\text { care, that a care service provider did that, but also because he wanted to have other } \\
\text { people around him and not just me. Very normal. }\end{array}$ \\
\hline
\end{tabular}

Based on the categorization of the data, we present detailed descriptions of our categories in the following section. The descriptions contain a short introduction and then go into more detail as we use the subcategories implicitly to structure the findings. Additionally, we added vivid examples or meaningful quotes of caregivers and relatives whenever they help to comprehend the circumstances in the care settings.

\section{Category-based Analysis for Intensive Home Care}

The following sub-sections are organized on the basis of the categories listed in Table 1 in the preceding Section 3.

\subsection{Activities in Intensive Home Care}

Activities characterize the tasks and the behavior of people in the field of intensive home care and its context. The differentiation of activities describes the behavior of the three main actors (caregivers, relatives and clients). It helps to understand the bigger picture of the work done in intensive home care. Mainly, it is important to differentiate between initial and regular tasks.

Usually, the first activity to be conducted at the start at a certain place is the client admission. It includes collaborative work (cf. Section 4.2) and talking with the client and relatives. Professional caregivers mainly have to elicit all essential information about the client's condition, handling, biography and medical history. Typically, relatives accompany the client's whole life of suffering and therefore have a detailed view about the client's problems and needs. Relatives are the first point of contact to receive client related information and to organize the further daily treatment in an intensive home care setting. Information gathering lasts until one is capable of providing the adequate treatment without further help from the relatives. There is no clear rule when this is completed.

The daily care routine consists of personal-care services (e.g. feeding, washing, etc.) and technical nursing (e.g. skin care, oxygen administration, etc.) [35]. It is important to notice that most of these activities have to be documented for the care service provider in detail (see Section 4.3). Furthermore, there are handover activities with the other caregivers, domestic-aid services (e.g. housekeeping, food preparation, etc.) related to the client (and not relatives), and organizational activities (e.g. scheduling appointments, picking up pharmaceuticals, etc.; see Section 4.2.i). In many cases the latter are carried out with the relatives mainly in a mutual way. In addition, relatives mainly carry out domestic activities, but in most cases are hardly or not involved at all in personal-care services and technical nursing for the clients [35]. Additionally, the client's activities are highly relevant since they try to manage their everyday life as far as possible depending on their health condition. Therefore, the caregiver's activities are influenced 
by the possible autonomy of the client, which makes the knowledge about the client's biography and medical history highly relevant, as it can influence the relationship between client and caregiver.

Handover activities are generally managed only between the caregivers, mainly because the information being exchanged concerns the caregiver's change of shift. The information helps to keep each other updated when something unusual happened compared to a 'normal' shift. Usually, relatives are not involved in handover activities, because information related to the client's treatment is normally documented in a specific, non-digital document folder which relatives may inspect anytime (see Section 4.3). On the other hand, there are situations that caregivers first have to discuss with their team leader or with the care provider. Typical topics are, for instance, a client's inappropriate behavior towards the caregivers or developing a suitable solution how to handle a critical situation together with relatives and the client.

Depending on the client's state of health, caregivers can be faced with (what we call) idle phases without immediate demand for work, e.g. caregivers are responsible for persistent vegetative state (PVS; see [36]) clients. Other caregivers hardly have those idle phases, and if so, they used them for care related activities like documentation or domestic tasks. Several interviewees reported that clients demand domestic activities within the intensive home care environment that were not agreed upon with the care service, such as cleaning up the living environment of the client's family, buying groceries or doing the laundry for the client's relatives.

\subsection{Interpersonal Interaction}

Interpersonal interaction characterizes the collaboration between the three main actors: caregivers, clients and relatives. We identified three dominant constellations where information exchange, coordination and conflict management are highly relevant. It can be hypothesized that relatives tend to leave the care activities to the caregivers (see Section 4.1). To understand the field of intensive home care we consider the interaction with third parties (vendors, physicians, physical therapists etc.) as less relevant, as influences by these roles were hardly observable or mentioned by the interviewees. The three dominant constellations are described in the following:

i) Interaction between professional caregivers and relatives is mainly focused on organizational tasks and less on care activities. As a possible reason we suggest that relatives simply felt overwhelmed with care activities and therefore value the increasing freedom they gain as soon as a professional care service is available. When asked whether relatives would also help with scheduled care tasks, one caregiver validated:

"Well, I never experienced that, because most cases were really that way that relatives relied totally on the care team, because they were glad that they didn't have to do it on their own." (Caregiver WBR06).

However, this new freedom is constrained since the uninterrupted presence of professional caregivers all the day has an impact on the relatives' privacy and might result in conflicts. Opinions of professional caregivers on the involvement of relatives range from caregivers who are convinced that relatives should not be allowed to conduct technical nursing activities, because they lack the adequate qualification, to caregivers who rather try to motivate relatives to take over some of the nursing activities. One of the main problems related to these circumstances are the legal liabilities regarding the client's health. In particular, something unforeseen during care activities might happen and, in worst case, the client may be harmed. Although these legal constraints are not always considered, care service providers are obliged to instruct caregivers about them. Another controversial subject is the fact that the responsibilities regarding the domestic activities are often not clearly communicated to each other. In several interviews, caregivers reported that some relatives think caregivers are, e.g. responsible for cleaning the whole living environment of the client, while the caregivers emphasized that they are only responsible for housekeeping activities related to the client. 
Furthermore, as already mentioned in Section 4.1, organizational activities tend to be carried out by caregivers and relatives mainly in a mutual way. Relatives seem to use the opportunity of relieving caregivers from and supporting them with certain organizational tasks to stay involved within the client's everyday life. Those tasks can include scheduling appointments with physicians, physiotherapists or therapists (e.g. via phone call), accompanying the client to the aforementioned appointments, ordering and/or retrieving (prescribed) pharmaceuticals, taking them to the pharmacist, doing the shopping for the client, etc. For instance, when asked about the support of relatives, one caregiver told us:

"For example, when the client has to be dressed. For this, I can ask the relatives to hand me over the clothes. [...] Or ordering prescriptions from the physician. All these [tasks] are done by relatives." (Caregiver DTR22).

Relatives point out that organizational tasks can be more easily conducted than activities such as personal-care services or technical nursing, especially at their work. The caregivers emphasize that although the collaboration on organizational activities works out quite well, they must rely on the relatives nevertheless, otherwise critical situations for the client can arise, for instance if an essential medication is not at hand.

ii) Interaction among professional caregivers. On the one hand, caregivers largely describe their collaboration as harmonious. On the other hand, the level of conscientiousness and accurateness is considered as very heterogeneous between the employees, and the client can be negatively affected because of these differences. A further type of problems is caused by the shift system that has to guarantee 24/7 care. For instance, an employee's absence caused by illness can result in scheduling and coordination problems. Further issues concern the handover phase: the caregivers complained multiple times that handover phases usually cut their free time, because the change of shift usually takes place after a shift has already been finished. Additionally, caregivers briefly discuss information about the client if something deviates from the everyday routine. Digital or written notes are hardly used for this exchange. Most of the time such verbal exchanges work well for the caregivers, but occasionally it can result in a loss of information. This lack of communication increases if caregivers are negligent about their documentation duties or if specific information about the client is not available for all caregivers. For instance, this is the case if information is only accessible via a computer that can only be used by the caregivers' team leader.

iii) Interaction between professional caregivers and clients. Professional caregivers' interaction with the client is faced with a trade-off challenge: on the one hand, they try to offer space and time for privacy, e.g. opportunities where a client can communicate privately with the relatives. On the other hand, the caregiver must always be ready to help the client and therefore has to monitor the interaction between the clients and their relatives.

Problems and conflicts in the interaction with the client can be caused by a lack of biographical information about the client. These problems can result in miscommunication or antipathy between caregivers and clients. An example are situations where clients schedule appointments with relatives and friends, but do not inform the caregivers about these events, thus giving them no possibility for preparing the client for these encounters.

\subsection{Documentation}

Documentation is either related to noting down information such as vital parameters, or it is a meta-activity in relation to other activities such as documenting a care task or communication with externals (physicians, service providers, physiotherapists etc.). In addition, there is also other information documented by caregivers that they consider relevant, e.g. deviations from the routine regarding the clients' nursing. The documentation is highly important for documenting the health status and vital parameters of clients, but also for providing evidence of service by the care service provider, as this evidence is needed for the payment they get from health insurance companies in Germany. Therefore, consistency and completeness are relevant criteria for 
documentation. Additionally, documentation is needed for quality checks. To document, caregivers have nursing context-related plans of tasks. Conducting appropriate documentation is challenged by time pressure, stress, forgetfulness or laziness, often depending on aspects like the client's health condition or the activities to be conducted within this context (see Section 4.1).

We identified various behavior patterns for documentation:

- One caregiver started the documentation only after finishing all her tasks and she memorized parameters for some time, leaving a risk to alter or forget certain information.

- Others just made pictures of the client's current vital parameters on the ventilation machine display to write them down into the documentation sheet afterwards (see also Section 4.5).

- Some caregivers reported that other team members simply forget to document vital parameters or other important information in general, thus causing further problems, e.g. related to the client's insurance billing.

- One caregiver reported about forgetfulness or inattention that is intertwined with idle phases without immediate demand for work (e.g. when working with PVS clients).

Various ways are employed to convey information that is relevant for documentation: Some caregivers document information as far as possible within the document folder, others exchanged information only verbally during the shift change in handover phases with the following caregiver. These caregivers potentially suffer from a lack of information, e.g. about a client's emotional condition.

\subsection{Qualification}

Qualification covers all kinds of knowledge and experience needed to carry out the specific tasks of intensive home care including technical nursing as well as social competence. It can be grouped into three subcategories: occupational history, further education, and introductory training.

Every interviewed caregiver is at least generally qualified as a nurse or as a caregiver for the elderly (in Germany: regular apprenticeship of 3 years). However, the interviewees differ widely in terms of their years of work experience and their practical experience, as the reflecting of their occupational histories reveals. For instance, years of work experience range from three to thirty. Practical experience was made in various areas. The interviewees reported that they worked in areas such as surgery, gynecology, intensive care units, and nephrology - ambulatory as well as stationary. Moreover, caregivers differ in their job positions and activities. Some of them do not exclusively work on the operational level but are also active as team leaders, team coordinators, and/or nursing managers.

With respect to further education and introductory training, we found that it seems possible for new staff to start working in areas like, e.g. home mechanical ventilation without any practical experience in intensive care, home care and/or ventilation. Although one caregiver told us that it is mandatory to pass a course of ventilation as a part of further education to work in intensive home care, one of her comments also indicates that this is typically not the case:

"Attending the [basic qualification] course, I was very upset that I was the only one [...] who was not already employed in intensive home care while doing the course. All the others were already active within this job." (Caregiver MAE16).

Inexperienced caregivers usually get a guidance for about one to three days in the client's environment, within which they are supposed to learn all essential tasks and activities including the necessary (nursing) technology usage. We assume that because of this limited time for instruction, inexperienced caregivers may be - or at least feel - insufficiently prepared to handle their tasks or to deal with complex technology. Insecurity can result from missing knowledge or routine. For instance, the endotracheal tube suction must be carried out very carefully to avoid 
injuries of the client's respiratory tract. Inexperienced caregivers learn this activity only with the help of anatomical torso models during their apprenticeship, which can cause a high level of insecurity. Another example relates to the replacement of utilized tracheostomy tubes: The client's health condition might be threatened due to contamination, if the caregiver's hands or the applied devices are not sufficiently sterilized.

We learned that especially technical nursing activities like the home mechanical ventilation or the endotracheal tube suction can be exceedingly complex, so that a lack of knowledge about the technologies related to them could result in life-threatening situations for the clients in worst case.

\subsection{Interaction with Technology}

'Interaction with technology' does not focus on the information system and collaboration support that is addressed by a potential information system solution but refers to the kind of basic technology that primarily supports the services to be carried out. Based on our material, we differentiate between four groups of technology: for personal-care services, for technical nursing, for organizational activities and for domestic-aid services.

Technology for personal-care services (e.g. assistance with dressing, feeding, washing and toileting, and getting in or out of bed etc.; see [35]) requires different kinds of interaction by caregivers and by relatives. The type of devices and their usage depends on and varies with the clients' health characteristics. Examples are simple devices like electric toothbrushes or more complex ones like specific modifications of certain rooms (e.g. bathrooms for easier handling of the client) or systems like lifters and electronic wheelchairs. Technology for technical nursing is mainly used to preserve the client's life and to conduct nursing activities. These technologies also strongly depend on the client's health condition and have to be used for activities like assisting to put on prostheses, changing stomas, helping with bladder catheters, disinfection, oxygen administration, or giving intravenous injections [35].

Technologies for organizational activities are usually used by caregivers as well as by relatives for communication to coordinate organizational tasks. Typically known from office environments, these devices comprise, e.g. desktop computers, laptops, printers, fax machines or smartphones. For instance, in some cases the communication was also done informally via messenger-apps among caregivers but also between caregivers and relatives. Technologies for domestic-aid services can be found in any other regular household; such as a simple (microwave) oven or cooker, or vacuum cleaner, etc. for typical activities like shopping, food preparation or housekeeping [35].

Our studies reveal that the most complex challenges arise from the interaction with technologies for personal-care services and technical nursing that are mainly used by professional caregivers. For instance, we observed a clear heterogeneity of knowledge about the clients' technical devices used for mobility. One caregiver told us that it is necessary to maintain an electric wheelchair regularly that is equipped with a mobile ventilation system. While experienced caregivers normally master the details of their devices, less experienced ones usually miss some necessary information. This deficit burdens the cooperation between them, because inexperienced caregivers simply leave this task to the experienced ones.

Various devices for ventilation are used in intensive home care. Even experienced caregivers in home mechanical ventilation reported that it sometimes happens that they are confronted with new devices for ventilation or have not been working with specific devices for a longer time. Although they have experience with devices for ventilation, they do not know how to handle every device. One caregiver suggested to provide information about how to handle devices and the related activities. 


\subsection{Client Autonomy}

Client autonomy defines the way in which caregivers expect autonomous handling of certain daily activities by the clients. We observed a contradiction about the autonomy of clients: On the one hand, they usually must get support for 24 hours a day and seven days a week, thus they depend on the care carried out by caregivers. On the other hand, dependent on client's health condition and degree of impairments, caregivers are supposed to encourage clients in carrying out everyday tasks autonomously, e.g. making appointments or ordering food.

From the caregivers' point of view, clients can become severely reliant on the support of caregivers. This dependency consequently can result in a lack or even total loss of the client's autonomy in worst case. Tasks like making appointments with physicians or getting groceries do not necessarily have to be delegated to the caregivers, but can also be conducted by clients. However, clients might try to dispose these tasks to the caregivers, because in their opinion, caregivers seem to have a lot of idle time apart from caregiving phases and, as they are seen as a part of the living environment, they consequently could carry out also client related activities. Nevertheless, we observed also clients, who try to live as autonomous as possible within the scope of their health condition, e.g. doing activities like going for shopping or doing the laundry. The demand for the client's autonomy was even more evident in situations of domestic activities: a client with advanced muscular dystrophy, was able to use only one arm with two fingers of all of his limbs, but nevertheless managed to order himself groceries or food via a laptop over the internet and therefore was responsible for this task on his own. This ability could also lead to further problems related to the coordination with his caregivers, if he wouldn't take care of it appropriately and, e.g. would not order groceries for himself. According to them, this could happen from time to time and result in conflicting situations, in which the caregiver was not able to provide the client with any food, because the client simply missed to inform his caregivers about this fact.

Moreover, in rare cases we observed clients who were able to expand their autonomy by conducting personal-care services and technical nursing activities, which we generally associate with caregivers in most cases (see Section 4.1). Again, this depends on their health condition and degree of impairments. For instance, regarding to personal-care services, we could watch a client cleaning her face after sleep or brushing her teeth after breakfast. Related to technical nursing activities we could observe a client with a tracheostoma connect an inhaler to it and consume a medicinal substance all by her own. Therefore, adaptive functionalities to the varying range of tasks that can be conducted by clients due to their health conditions is a crucial challenge for the development of an information system. Such an IS should not only be able to store and display information about the disposition of tasks, but should also be adaptable to every individual care setting and client's state. Furthermore, the information on this IS should be quickly and easily retrievable, as especially new caregivers or ones, who change their workplace very often, must be able to efficiently obtain required information to run the activities that are related to the client. Compared to settings like intensive care units, intensive home care is a long-term oriented support. Therefore, an information system has to allow and support the individual configuration of information that is needed for the care setting. This also includes the adjustments that might be needed due to health changes of a client.

As a result, the IS should offer lists of typical tasks. These lists should be adaptable by both the caregivers as well as the clients, and allow for the coordination of task sharing between them which can be configured flexibly. The development of the client's autonomy or increasing dependency should be documented to support reflection and to foster his/her autonomy during everyday life. Not only the tasks being carried out by the caregivers but also those ones being conducted by the clients and accompanied by the caregiver's assistance or supervision have to be appropriately documented. 


\subsection{History of Medical Records}

This category contains information about the relevance of individual medical records and biographies of the clients. We divided this category into three sub-groups: biographical information about the client, relatives' burdens, and relatives' self-help related to the client's care. According to the reports of our interviewees about the clients' individual paths of life, a lot of effort has to be summoned up to understand and consider the medical records of clients, their family background, as well as rituals and personal preferences in specific care situations. Caregivers mentioned that biographical information like rituals and preferences should be written down in the documentation folder as soon as a caregiver finds the specific preference noteworthy. One caregiver told us about a case, in which a client with a feeding tube was physically not able to feed herself. Although the individual impairment was noted, the caregivers did not write down how the situation could be solved in everyday situations with the client:

"Between eleven and fourteen o'clock I read in my schedule: $<<$ Likes to drink a half cup of coffee. >> Ok, but holding it in her hands is not possible. If I put the cup at her mouth, it pours out, because the given amount can't be swallowed. So I wrote down additionally: "How?" While many colleagues made 20000 question marks before, because everything else is only possible over the tube, no matter if it is medication or water [...]. Then I found out that her husband [...] sweetens a cup of coffee with honey and puts little doses of coffee into her mouth with a tea spoon. Well, this would have been an information I could have used. " (Caregiver SRH19)

Furthermore, the caregiver also mentioned that the client hyperventilated when trying to give her coffee in other ways, which, again, shows the importance of documented preferences as well as the instructions on how to solve similar situations.

From such reports, we derive that an IS should be able to support the communication process about lacks of clarity in similar cases within a (more) narrow time frame on the one hand. On the other hand, it needs to provide biographical information as quickly as possible in specific care situations. For this, the IS must help to understand the clients' biography and conditions as a continuous transition that should be comprehensible. Therefore, not only isolated situations but a sequence of changes during long-term care has to be documented.

\subsection{Feedback on Intensive Home Care by Relatives and Caregivers}

During our data acquisition, caregivers as well as clients mentioned positive and negative statements and ratings about intensive home care, that are all subsumed in this category. Whereas the relatives of clients generally appreciate intensive home care as very helpful and as a relief, the caregivers' opinion was more diverse. As an example, long working shifts were rated positively because it leaves caregivers the chance to reduce the number of total workdays per year. On the opposite, these long working days were classified as time consuming and adverse to free time, thus, e.g. making it more difficult to reserve time for the family. Furthermore, some caregivers also mentioned being sometimes criticized by the clients' relatives during caregiving activities, e.g. because relatives did not like the way the caregivers conducted certain tasks. This criticism resulted in disturbed social interactions during care and made their work even more difficult.

Although working in intensive home care was also rated as very positive in general by many caregivers, especially due to the more intense supplying character for individual clients compared to other care settings like intensive care units, the number of negative comments on various topics was much broader. For instance, concerns were mentioned about the introduction into intensive home care without an adequate education in intensive care. This deficit can cause a high degree of insecurity especially among new caregivers (see Section 4.4). Thus, caregivers expressed a need of having access to further educational material and instructions about specific 
technical nursing techniques like wound treatment, the procedure to change a cannula, or the handling of ventilation machines. Another important (negative) topic that was mentioned quite often referred to staff shortage: Multiple caregivers complained about the lack of available temporary employees, who can deputize a staff member e.g. in cases of illness that occur quite often within this field.

Finally, we asked our interviewees about ideas, wishes, and improvement proposals for their work in intensive home care. In summary, the following issues were mentioned:

- The lack of access to information about the client was one of the most criticized problems,

- Caregivers asked for faster and easier information retrieval, and less searching of paper documentations,

- Technical reminders to prevent forgetfulness,

- Guidance videos about specific tasks,

- Improved information exchange during handovers.

\section{Comparison of Results to Related Work}

To relate our ethnographically derived results to other research in the health care domain, we have compared our results to the concluding chapter of the book "Designing Healthcare That Works" by Ackerman et al. [5]. Derived from 10 case studies [29], the chapter discusses sociotechnical consequences for the health care domain that we consider for the comparison with the results of our fieldwork and examine how the general statements apply to the special health care domain of intensive home care.

\subsection{Social Arrangements and Interaction}

Ackerman et al. emphasize an increasing complexity of socio-technical systems in the health care domain, as health care involves complex social arrangements. Besides the medical staff, social arrangements can also include family caregivers or home-helpers [37]. They mention a reorganization of formal and informal roles in health care, which we could not observe in intensive home care. In comparison, although our ethnographic study confirms the complexity between involved parties, we did not observe family caregivers. On the contrary, caregivers have assured us that family members felt relieved, when the care service provider had taken over the care of clients (see Section 4.1 and 4.8). Relatives did, however, try to help with organizational tasks (e.g. setting appointments with physicians or retrieving prescribed pharmaceuticals) but less with caregiving activities. Moreover, the interplay between roles and their task sharing does include all kind of other activities that are related to everyday life such as housework, social entertainment, organizational issues, etc.

Social interaction in the domain of health care also integrates translation work between medical professionals and patients [38]. In our study we found that caregivers are always the first contact for relatives for understanding the medical need of certain treatments or a general understanding of medical information. Aside from their all-day presence, caregivers tried to stay in the background as much as possible, leaving clients and their relatives as much space as possible. However, caregivers are depending on a good functioning relationship within families for a healthy social climate in the home care settings, for instance, they rely on the willingness of relatives to support the aforementioned organizational tasks or others.

Compared to the highly dynamic health care domain [29], intensive home care settings can be viewed as rather static for most of the time, as a team of caregivers is usually assigned for one client. However, these settings can change rapidly and get dynamic, if, for instance, the health conditions of a client change or the illness of caregivers cannot be compensated within the team. In the latter case, external caregivers must step in or the client has to be transferred to an intensive care unit temporarily. This example supports the suggestion by Ackerman et al. that the 
characteristics of physical locations where technology is employed are of high relevance [29], as the intensive home care setting might include external staff or change the clients' location temporally.

Another central point for socio-technical design in health care is understanding the processes and workflows between patients and clinicians. For instance, Ackerman et al. argue that the design process should analyze and understand the as-is-processes. Here, technically supported health care should not only focus on efficiency, but should also support a more holistic view considering patients' health development and their trajectories. Even their care networks and social environments may continuously change. We could also find the relevance of medical histories and changing life circumstances in our data. The categories 'client autonomy' and 'history of medical records' show that the health status of clients changes over time and can have a strong impact on the autonomy in their lives. The time-orientation should incorporate clients' trajectories as care settings or services can change. Still, many tasks in intensive home care are done in structured workflows, like technical nursing tasks or routines of personal-care services. Additionally, each client has his/her own day's schedule that is processed by caregivers.

\subsection{Motivation and Values}

Mol [4] argues that socio-technical design in health care has to understand the underlying logics driving people's work, as well as the organizational or institutional cultures. Our results suggest that every activity that goes beyond the formal duties of the caregivers depends on their value system (e.g. challenging clients to take care of themselves and to live healthier). Additionally, Ackermann et al. argue that there are also more monetary oriented "logics of accounting and efficiency motivating parts of health care information technology [29, p. 189]. For intensive home care, this applies predominantly to the usage of the core medical technology and less to communication and information processing support.

Ackermann et al. mention an intersection of interests [39], which can support or oppose each other. Therefore, flexibility is needed to address different logics. In our study, we were able to observe this need on several levels. For instance, legal issues have to be solved when relatives want to provide technical nursing. In such situations the improper handling of material can harm clients (e.g. changing a tracheal tube). Organizational examples can be found in the specific environments of households: caregivers have to negotiate with clients and relatives where technical equipment may be located. Here, for instance, cables might lie across rooms as stumbling hazards, but are necessary for the machines observing the clients' health condition.

In the health care sector, increasingly the question has to be answered whether processes and involved decisions should be automated or whether these decisions should be reserved for people. Ackermann et al. suggest that an appropriate answer is only possible if one knows for who one designs the system, who is affected by it and how one considers the values of the affected persons. This problem is addressed by the literature as well as by our study. If vital parameters, like, e.g. the pulse, will be saved automatically, an additional gap between caregivers and clients may arise, since documenting these parameters manually is an occasion to initiate interpersonal communication. As health care has shifted its focus more on life goals instead of mere health care goals [5], our study reveals that caregivers tried to not only maintain the health condition of clients. Caregivers also supported clients to maintain their social life (e.g. by motivating them to invite friends) or tried to fulfill personal preferences and wishes (e.g. serving sweetened coffee). Socio-technical design has to support the needs and values shared by individuals and families to gain trust and social coherence [5]. This was also reflected in our results. In a more problematic case, a caregiver reported that a client refused personal and technical care from most caregivers, except the ones who had won the client's trust. With biographical work it turned out that she was misdiagnosed several times and that these experiences led to a lack of trust to most caregivers. This example shows how important trust is for care settings. 


\subsection{Health Care Data and Technology}

Another important aspect for a socio-technical system in health care is the tracking of healthrelated data that, in addition, might log vital parameters automatically. This should not neglect the fact that in intensive home care a lot of documentation is still carried out manually. For instance, caregivers also document the overall condition and feeling of clients from their point of view. Ackermann et al. state that "clinicians are often concerned with their workflow" [29, p. 190] and attention to data would require additional effort. It is questionable if caregivers (or others) would reflect on the client's health condition among such complete trajectories of healthrelated data.

However, new applications and tools that record and save patients' data will arise. Historically, health care has passed strong changes in the past. When, at first it focused on medical instruments, it changed to many other types of support like health applications, social networks or other communication systems. Our results reveal that especially organizational issues between caregivers, as well as between relatives and caregivers, are communicated via messenger apps. Problematically, such apps do not necessarily meet all privacy requirements. Therefore, a socio-technical analysis should also include the analysis of "self-established" technical support in the domain. Besides messenger apps, we did not find other apps that were used for health-related data. However, if such apps/systems should be used, medical machines would need proper interfaces (with respect to privacy aspects) to import and export healthrelated data for other health-applications. Our results have shown, for instance, that a system supporting the documenting and saving of clients' vital parameters at several points a day could improve the current documentation procedure. Some caregivers tried to memorize parameters until they finished providing technical nursing. Here, a logging system could prevent the risk of forgetting the correct parameter values. A solution could, for instance, log data temporally, so that caregivers could focus on their workflows before they have to document vital parameters.

\subsection{Challenges and Problems for Socio-technical Design}

Ackermann et al. describe that complex social structures, interactions and missing transparency can lead to people not always understanding everything that happens in health care. Here, sociotechnical design tries to dissolve this by incorporating the complex and maybe unstructured processes instead of minimizing its view on rule-based workflows. In our study, the ethnographic approach revealed different strategies of caregivers to solve tasks by implementing their strategies into existing routines. Besides helping to understand their work, the approach opened up requirements for a potential socio-technical system that should take such workarounds or routine-modifications in account. A vivid example of such a workaround is, that caregivers took pictures of machine displays with their smartphones to save snapshots of vital parameters, when they were providing technical nursing. This way, they could document the parameters afterwards. If, for instance, this manual documentation of vital parameters would be changed to an automatic data logging, Ackermann et al. point to challenges that might occur with a permanent and continuous data logging. If electronic systems save and log this data continuously, most parts of it will probably never be analyzed. In addition, it brings the risk that physicians might lose themselves in too many details instead of building up a holistic view over the patients' situation. Simultaneously it increases the risk of violating privacy.

Ackermann et al. speak of a possible mistrust of patients and physicians concerning the design of technical systems. This might refer to privacy aspects and is related to a lack of transparency. Our data has shown that clients and relatives had always had the possibility to examine the (physical) documentation files. Caregivers stated that the care service providers try to keep their work transparent for clients and relatives. Such demands have to be incorporated into a sociotechnical system if mistrust should be prevented, especially as information systems are harder to comprehend compared to physical documentation files. 
Finally, Ackerman et al. discuss in several chapters the lack of willingness to use new applications or technology. Some tools might be used differently than for what they were designed for [40] or might be used only for a short phase directly after their implementation but not continuously. During our study, we observed the usage of messenger-apps by relatives and caregivers to communicate informally. From a socio-technical view, a design has to offer at least alternative apps that offer, from a legal point of view, enough technical measures to prevent the violation of privacy (e.g. because of unencrypted data stored on application servers). This applies to messenger-apps as well as to the data capturing of vital parameters with smartphone cameras. Here, a clear willingness to use such tools already exist, but appropriate solutions were missing. Therefore, acceptance to use a supporting system would probably exist, as the need for such a snapshot-function was apparent and the established workaround is not compatible with medical data privacy. Another example in our study has shown how caregivers tend to skip technologyrelated tasks, if they do not feel confident enough to fulfill them. For instance, one caregiver told us that some of his/her colleagues do not do the maintenance of wheelchairs or oxygen bottles. Here, a systematic workflow-oriented guidance could support caregivers to fulfill these tasks. Even if caregivers would use such systems only rarely or until they feel confident enough to do the task on their own, such a guidance could support and help during training.

\section{Conclusions}

Intensive home care can be characterized as a complex socio-technical system that is shaped by the social interaction between three main parties: caregivers as members of caregiving institutions, clients and relatives. All three of them potentially undergo continuous transitions with respect to their capabilities, attitudes, preferences, and willingness to share certain tasks. Consequently, intensive home care has to be understood as a highly dynamic field.

Our studies reveal that employing information and documentation systems in this field for the purpose of accounting and contributing to health record systems is not the main purpose. By contrast, providing guidance for the caregivers and supporting the interaction between the three types of participants is additionally of high relevance, if ensuring the wellbeing of the client with affordable effort is considered as a primary goal. Therefore, various purposes of documentation have to be considered:

1. Accounting and proof of compliance with legal regulations,

2. Handover activities between shifts, which support the information exchange between caregivers,

3. Coordination between the participants and support of flexibility under various constellations (including the task distribution and the degree of task completion, as well as spontaneous rescheduling if, for instance, caregivers fall ill),

4. Comprehensibility of the clients' biography and preferences including details and their context, as well as clients' ability and willingness to be autonomous,

5. Feedback and support of self-evaluation; the development of the clients' situation must potentially be a subject of reflection,

6. Support of continuous improvement and adaptation to changing situations.

The requirements for the first of these purposes (no. 1) are highly regulated to achieve completeness and reliability, and error prevention is of extreme importance. By contrast, the other purposes (nos. 2-6) can include informality and incompleteness.

Feasible functionalities of an IS can be a lightweight capturing of information and the possibility to transfer this captured data into the documentation. Various types of hardware to capture or to display data have to be supported. The application of various eligible devices like smart glasses, tablets or PCs may be of different feasibility with respect to the task or the location where the caregiver is active. However, these diverse constellations must not lead to heterogeneous media being used and between which data has to be transferred and conveyed into 
different formats. Information exchange and documentation should be seamlessly integrated with the medical devices and technology that has to be employed for intensive home care. We realize a huge gap between theoretically possible data integration and practical reality. Thus, we assume, that information systems have to provide temporary solutions to bridge this gap before such a data integration is completely brought into reality.

Closely related to documentation, the system should also provide guidance with respect to the tasks that have to be carried out by caregivers. This guidance has to compensate the heterogeneous levels of experience and qualifications and task performance. Therefore, documentation has to provide a fluent transition to learning support. The more irregularly or occasionally the challenges have to be met, e.g. when dealing with medical devices, the more guidance seems to be necessary. Furthermore, guidance can also be important for relatives, for instance, information about duties and liability has to be available. Apparently, the type and extent of information displayed has to be highly adaptive with respect to situations and people's knowledge, experience, and interest.

One of the latest case studies published in our context is Ackerman's et al. [30] report on the challenges of providing home care for patients with spinal injuries. The similarity in the findings is that the relevant tasks and measures to support the client cannot be a subject of rule-based guidance. Although the empirical study is focused on Germany, the work of Ackerman implies that our findings also cover the situation of other countries. The difference to our field of intensive home care is that caregivers can have potentially more idle time and that the relatives have only a minor or even no official role with respect to contributing to the care of the client's health. However, to contribute to her or his wellbeing is also an important task for relatives. This contribution is not formally regulated, but is usually negotiated, for instance, with respect to the task sharing between the caregiver and the relatives. The need to support this negotiation and the informal ways of coordination are new findings compared with Ackerman's et al. study [30].

We suggest that the more time the professional caregiver is involved at a certain place, the more a specific social setting evolves with its own conventions, values, and goals to be pursued. With respect to this setting, the influence of goals of other stakeholders, such as managers or doctors, are more of minor relevance. The decisive goals are implicitly encoded in the daily behavior routines of such a setting and are not explicitly documentable as, for instance, expected by the $i^{*}$ [28] method. The goals vary in relation to the clients situation (e.g. from helping people help themselves to complete caretaking). Consequently, it would be necessary for the $i^{*}$-notation to combine goals with vague conditions whether and when they are instantiated or not. Therefore, it seems to be more feasible that requirements elicitation in this field should be based more on understanding the behavior patterns and routines of the involved people and the dynamics of their characteristics or preferences, and less rely on descriptions of goals or intentions.

Considering the used methodological approach, we identified some limitations. For instance, whereas the approach of GT provides a very detailed picture about the domain of interest, it is a time consuming process for the identification of requirements for health care information systems. Although this detailed view is based on multiple, individual care situations and interviews, the method and the sample size might limit the generalization of findings. However, the combination of observations and interviews allows not only to draw detailed conclusions about the work in the domain of intensive home care, but also provides a broader view on the domain through the comments of caregivers that allowed to confirm situations that have been observed as common. Comments of a couple of interviewees confirm that some situations we have observed are of a more common nature and do not only refer to intensive home care. It should also be mentioned that the interviews and observations were conducted by only three researchers in total and may be limited by their subjective view. This researcher bias does not only refer to the data collection process, but also includes the analysis. If the data were analyzed by other researchers, the category scheme could also be structured differently. 


\section{References}

[1] J. Kamaroddin, "Requirements Engineering for Pervasive Healthcare Monitoring System," Workshop Proceedings, CEUR, vol. 1564, 2016.

[2] L. P. A. Simons and W. P. Verhagen, "Applying value-sensitive design and quality function deployment to healthcare ICT: the case of Dutch primary care unit dossiers," J Des. Res., vol. 7, no. 2, p. 155-176, 2008. Available: https://doi.org/10.1504/JDR.2008.020853

[3] C. Keller, Fachpflege Außerklinische Intensivpflege. München: Urban \& Fischer Verlag/Elsevier GmbH, 2017 (in German).

[4] A. Mol, The Logic of Care: Health and the Problem of Patient Choice. Routledge, 2008. Available: https://doi.org/10.4324/9780203927076

[5] M. S. Ackerman, S. P. Goggins, T. Herrmann, M. Prilla, and C. Stary, Eds., Designing healthcare that works: a sociotechnical approach. London: Elsevier, AP Academic Press, 2018.

[6] I. Sommerville, T. Rodden, P. Sawyer, R. Bentley, and M. Twidale, "Integrating ethnography into the requirements engineering process," Proceedings of IEEE International Symposium on Requirements Engineering, pp. 165-173, 1993.

[7] M. Jelonek and M. Prilla, "Motivational Aspects of Using Augmented Reality Glasses in Care," Mensch und Computer 2016 - Workshopband, 2016. Available: https://doi.org/10.18420/muc2016-ws02-0004

[8] J. Hughes, J. O'Brien, T. Rodden, M. Rouncefield, and I. Sommerville, "Presenting ethnography in the requirements process," pp. 27-34, 1995. Available: https://doi.org/10.1109/ISRE.1995.512543

[9] C. Niemöller, D. Metzger, and O. Thomas, "Design and Evaluation of a Smart-Glasses-based Service Support System," Wirtsch. 2017 Proc., Jan. 2017.

[10] M. C. tom Dieck, T. Jung, and D.-I. Han, "Mapping requirements for the wearable smart glasses augmented reality museum application," J. Hosp. Tour. Technol., vol. 7, no. 3, pp. 230-253, 2016. Available: https://doi.org/10.1108/JHTT-09-2015-0036

[11] G. Baxter and I. Sommerville, "Socio-technical systems: From design methods to systems engineering," Interact. Comput., vol. 23, no. 1, pp. 4-17, 2011. Available: https://doi.org/10.1016/j.intcom.2010.07.003

[12] B. Lindberg, C. Nilsson, D. Zotterman, S. Söderberg, and L. Skär, "Using Information and Communication Technology in Home Care for Communication between Patients, Family Members, and Healthcare Professionals: A Systematic Review," Int. J. Telemed. Appl., vol. 2013, article 461829, 2013. Available: https://doi.org/10.1155/2013/461829

[13] J. Wiles, "Informal caregivers' experiences of formal support in a changing context," Health Soc. Care Community, vol. 11, no. 3, pp. 189-207, 2003. Available: https://doi.org/10.1046/j.1365-2524.2003.00419.x

[14] T. Bratteteig and I. Wagner, "Moving Healthcare to the Home: The Work to Make Homecare Work," ECSCW 2013: Proceedings of the 13th European Conference on Computer Supported Cooperative Work, Springer, pp. 143-162, 2013. Available: https://doi.org/10.1007/978-1-4471-5346-7_8

[15] D. Challis, R. Darton, J. Hughes, K. Stewart, and K. Weiner, "Intensive care-management at home: an alternative to institutional care?" Age Ageing, vol. 30, no. 5, pp. 409-413, 2001. Available: https://doi.org/10.1093/ageing/30.5.409

[16] L. R. Christensen and E. Grönvall, "Challenges and opportunities for collaborative technologies for home care work," ECSCW 2011: Proceedings of the 12th European Conference on Computer Supported Cooperative Work, Springer, pp. 61-80, 2011. Available: https://doi.org/10.1007/978-0-85729-913-0_4

[17] E. Grönvall and S. Lundberg, "On Challenges Designing the Home as a Place for Care," Pervasive Health, Springer, pp. 19-45, 2014. Available: https://doi.org/10.1007/978-1-4471-6413-5_2

[18] M. Tixier and M. Lewkowicz, "Counting on the Group: Reconciling Online and Offline Social Support among Older Informal Caregivers," Proceedings of the 2016 CHI Conference on Human Factors in Computing Systems, pp. 3545-3558, 2016. Available: https://doi.org/10.1145/2858036.2858477

[19] M. Muller, "Curiosity, Creativity, and Surprise as Analytic Tools: Grounded Theory Method," Ways of Knowing in HCI, Springer, pp. 25-48, 2014. Available: https://doi.org/10.1007/978-1-4939-0378-8_2

[20] S. Viller and I. Sommerville, "Social analysis in the requirements engineering process: from ethnography to method," IEEE International Symposium on Requirements Engineering, Proceedings, pp. 6-13, 1999.

[21] J. A. Hughes, D. Randall, and D. Shapiro, "Faltering from Ethnography to Design," Proceedings of the 1992 ACM Conference on Computer-supported Cooperative Work, pp. 115-122, 1992. Available: https://doi.org/10.1145/143457.143469

[22] M. Schorch, L. Wan, D. W. Randall, and V. Wulf, "Designing for those who are overlooked: Insider perspectives on care practices and cooperative work of elderly informal caregivers," Proceedings of the 19th ACM Conference on Computer-Supported Cooperative Work \& Social Computing, pp. 787-799, 2016. Available: https://doi.org/10.1145/2818048.2819999

[23] L. M. Cysneiros, "Requirements engineering in the health care domain," Proceedings IEEE Joint International Conference on Requirements Engineering, pp. 350-356, 2002. 
[24] I. D. Sorby, L. Melby, and O. Nytro, "Characterising cooperation in the ward: framework for producing requirements to mobile electronic healthcare records," Int. J. Healthc. Technol. Manag., vol. 7, no. 6, pp. 506521, 2006. Available: https://doi.org/10.1504/IJHTM.2006.010430

[25] M. R. McGee-Lennon, "Requirements Engineering for Home Care Technology," Proceedings of the SIGCHI Conference on Human Factors in Computing Systems, pp. 1439-1442, 2008. Available: https://doi.org/10.1145/1357054.1357279

[26] J. L. Mate and A. Silva, Requirements engineering for sociotechnical systems. IGI Global, 2005. Available: https://doi.org/10.4018/978-1-59140-506-1

[27] E. S. Yu, "Social Modeling and i*," Conceptual Modeling: Foundations and Applications: Essays in Honor of John Mylopoulos, Springer, pp. 99-121, 2009. Available: https://doi.org/10.1007/978-3-642-02463-4_7

[28] E. S. Yu and J. Mylopoulos, "Understanding 'Why' in Software Process Modelling, Analysis, and Design," Proceedings of the 16th International Conference on Software Engineering, pp. 159-168, 1994.

[29] T. Herrmann, M. S. Ackerman, S. P. Goggins, C. Stary, and M. Prilla, "Designing Health Care That WorksSocio-technical Conclusions," Designing Healthcare That Works, Elsevier, pp. 187-203, 2018. Available: https://doi.org/10.1016/B978-0-12-812583-0.00011-0

[30] M. S. Ackerman, A. G. Büyüktür, P.-Y. Hung, M. A. Meade, and M. W. Newman, "Socio-technical Design for the Care of People With Spinal Cord Injuries," Designing Healthcare That Works, Elsevier, pp. 1-18, 2017. Available: https://doi.org/10.1016/B978-0-12-812583-0.00001-8

[31] J. Corbin and A. Strauss, Basics of qualitative research (3rd ed.): Techniques and Procedures for Developing Grounded Theory. Thousand Oaks, CA: Sage Publications, Inc., 2008. Available: https://dx.doi.org/10.4135/9781452230153

[32] V. Jupp, The SAGE Dictionary of Social Research Methods. SAGE, $2006 . \quad$ Available: https://doi.org/10.4135/9780857020116

[33] U. Kuckartz, T. Dresing, S. Rädiker, and K. Stefer, "Qualitative Evaluation. Der Einstieg in die Praxis," VS Verl Für Sozialwissenschaften, vol. 2, 2008 (in German).

[34] U. Kuckartz, Qualitative Inhaltsanalyse. Methoden, Praxis, Computerunterstützung. 3., überarbeitete Auflage. Weinheim: Beltz Juventa (Grundlagentexte Methoden), 2016 (in German).

[35] N. Genet, B. Wienke, M. Kroneman, A. Hutchinson, and R. B. Saltman, Home Care across Europe - Current structure and future challenges. United Kingdom: European Observatory on Health Systems and Policies, 2012.

[36] B. Jennett, “The vegetative state,” J. Neurol. Neurosurg. Psychiatry, vol. 73, no. 4, p. 355-357, 2002. Available: https://doi.org/10.1136/jnnp.73.4.355

[37] K. Abru Amsha and M. Lewkowicz, "Supporting Collaboration to Preserve the Quality of Life of Patients at Home - A Design Case Study," Designing Healthcare That Works, Elsevier, 2018, pp. 39-56. Available: https://doi.org/10.1016/B978-0-12-812583-0.00003-1

[38] E. Kaziunas, M. S. Ackerman, and T. C. E. Veinot, "Localizing Chronic Disease Management: Information Work and Health Translations," Proceedings of the 76th ASIS\&T Annual Meeting: Beyond the Cloud: Rethinking Information Boundaries, vol. 50, no. 1, pp. 1-10, $2013 . \quad$ Available: https://doi.org/10.1002/meet.14505001090

[39] C. Bossen, "Socio-technical Betwixtness: Design Rationales for Health Care IT," Designing Healthcare That Works, Elsevier, pp. 77-92, 2018. Available: https://doi.org/10.1016/B978-0-12-812583-0.00005-5

[40] J. E. Bardram and M. M. Frost, "Double-Loop Health Technology: Enabling Socio-technical Design of Personal Health Technology in Clinical Practice," Designing Healthcare That Works, Elsevier, pp. 167-186, 2018. Available: https://doi.org/10.1016/B978-0-12-812583-0.00010-9 